\title{
Psychologische Flexibilität messen: Der Fragebogen zu Akzeptanz und Handeln II
}

\author{
Jürgen Hoyer Andrew T. Gloster \\ Institut für Klinische Psychologie und Psychotherapie, Technische Universität Dresden, \\ Deutschland
}

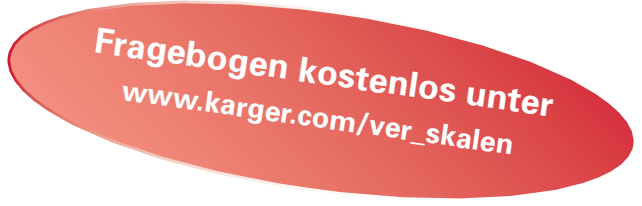

\section{Einsatzgebiet des Fragebogens zu Akzeptanz und Handeln II}

Der Fragebogen zu Akzeptanz und Handeln II [Bond et al., 2011] dient der Erfassung des Konstrukts der Psychologischen Flexibilität (PF) [Hayes et al., 1999]. Der Einsatz des Verfahrens ist bei der Evaluation von psychotherapeutischen Maßnahmen zu empfehlen, wenn über die Symptomreduktion hinaus auch die Entwicklung spezifisch definierter psychologischer Kompetenzen (im Sinne der Akzeptanz von psychischen Symptomen und der Handlungsfähigkeit trotz dieser) erfasst werden soll.

\section{Theoretischer Hintergrund und Kurzbeschreibung des Verfahrens}

PF ist ein zentrales Konstrukt im Rahmen der Akzeptanzbasierten Verhaltenstherapie (Acceptance and Commitment Therapy, ACT) [Hayes et al., 2012]. PF umfasst die «Fähigkeit, als bewusster Mensch in umfassenderer Weise zum gegenwärtigen Augenblick in Kontakt zu treten, wobei das Verhalten, jeweils der konkreten Situation entsprechend, entweder beibehalten oder verändert wird, um als wertvoll eingeschätzte Ziele zu erreichen» [Luoma et al., 2009, S. 39]. Damit ist ein «Konstrukt höherer Ordnung» gemeint, denn es integriert verschiedene Teilaspekte von ACT wie Erlebensvermeidung (experiential avoidance), Akzeptanz, kognitive Defusion und Achtsamkeit (mindfulness) (zu diesen Begriffen siehe z.B. Eifert [2011]). «Psychologisch flexibel» wäre es etwa, trotz schmerzlicher Erfahrungen und Erinnerungen das eigene Leben wertschätzen zu können (vgl. Item 1 des Fragebogens unter www.karger.com/ver_skalen) oder Gefühle/Sorgen je nach Situation unterschiedlich zu nutzen und zu bewerten, anstatt sie einseitig als Bedrohung zu erleben (vgl. Items
2, 5, 7 des Fragebogens). Es geht also nicht nur um die «Akzeptanz» von seelischen Belastungen, sondern auch um die Fähigkeit, trotz dieser und mit diesen Belastungen flexibel «handeln» zu können.

Nach Kashdan und Rottenberg [2010] beschreibt PF einen spezifischen Aspekt seelischer Gesundheit, den andere, gut untersuchte Konstrukte wie Symptomfreiheit oder psychologisches Wohlbefinden nicht abdecken. Damit ist das Konstrukt von allgemeiner Bedeutung und nicht nur im Kontext der ACT-Theorie relevant. Eine für die Psychotherapietheorie der ACT wichtige Annahme bezieht sich darauf, dass PF einen Mediator für die Veränderung (oder Bewältigung) psychopathologischer Symptome darstellt. Die Steigerung der PF wäre damit der Symptomreduktion vorgeordnet.

Weil es bei PF um Verhalten in Situationen geht, ist das Merkmal eigentlich verhaltensdiagnostisch zu erfassen. Um PF einfach und ökonomisch erheben zu können, gibt es Versuche, das Merkmal auch mittels Fragebogen zu operationalisieren. Der Acceptance and Action Questionnaire I (AAQ-I) [Hayes et al., 2004] ist ein (je nach Version) 9- oder 16-ItemFragebogen mit 7-stufigen Aussagen, die indikativ für PF sein sollen. Inzwischen wurde eine neue Version entwickelt, um insbesondere die interne Konsistenz zu steigern. Der AAQ-II [Bond et al., 2011] umfasst lediglich 7 siebenfach gestufte Items, die aufzusummieren sind. Der Wertebereich liegt damit zwischen 7 und 49, wobei höhere Werte höhere Inflexibilität kennzeichnen.

Die deutsche Version des AAQ-II, der Fragebogen für Akzeptanz und Handeln II (FAH-II), wurde im Rahmen einer Übersetzungs-Rückübersetzungsprozedur erstellt [Gloster et al., 2011a]. Der Fragebogen ist sehr gut handhabbar, die Bearbeitungszeit liegt bei wenigen Minuten. Inzwischen liegen Daten und Referenzwerte von mehreren klinischen (Panik-Patienten, Sozialphobie-Patienten) und Gelegenheitsstichproben (Studenten, Arbeitslose) mit mehr als 1100 Probanden vor.

\section{KARGER \\ Fax +497614520714 \\ Information@Karger.com}

www.karger.com (c) 2013 S. Karger GmbH, Freiburg

1016-6262/13/0231-0042\$38.00/0

Accessible online at:

www.karger.com/ver
Prof. Dr. Jürgen Hoye

Klinische Psychologie und Psychotherapie

Technische Universität Dresden

Hohe Straße 53, 01187 Dresden, Deutschland

hoyer@psychologie.tu-dresden.de 
Die angenommene 1-Faktor-Struktur ließ sich für alle Stichproben replizieren. Die interne Konsistenz (via RaykovEstimation) war gut ( $\alpha=0,84$ bei Sozialphobie-Patienten) bis sehr gut ( $\alpha=0,97$ bei Studenten). Die Test-Retest-Reliabilität nach einem 2-Wochen-Intervall war bei Studenten hoch $\left(r_{t t}=0,85\right)$, bei Panik-Patienten einer Warteliste lag sie bei $r_{t t}=0,75$ (erste und zweite Messung; 4-Wochen-Intervall) bzw. bei $r_{t t}=0,74$ (zweite und dritte Messung; 5-Wochen-Intervall). Konsistent über verschiedene Stichproben korrelierte PF erwartungskonform negativ mit Indikatoren von Depression (zwischen $r=-0,51$ und $r=-0,73$ ), Angst und Stress (zwischen $r=-0,50$ und $r=-0,59$ ), allgemeiner Symptombelastung $(r=-0,72)$ und Neurotizismus $(\mathrm{r}=-0.63)$ sowie Verhaltenshemmung $(\mathrm{r}=-0,58)$. Positiv korrelierte PF mit dem Selbstwert $(r=0,67)$ und mit Extraversion $(r=0,49)$.

Die für die Konstruktvalidität zentrale Annahme, dass PF zur Erklärung der psychischen Funktionsfähigkeit einen über Neurotizismus, Angst und Depression hinausgehenden inkrementellen Beitrag leistet, fand sich innerhalb der Stichprobe der Sozialphobie-Patienten bestätigt. Für Panik-Patienten ergab sich hinsichtlich der Funktionsfähigkeit ebenfalls eine bessere Varianzerklärung, wenn PF als Prädiktor berücksichtigt wurde. Dies galt aber nicht für die Erklärung agoraphobischen Vermeidungsverhaltens. Dass PF tatsächlich einen Mediator für nachfolgende Veränderungen im Bereich der psychischen Funktionsfähigkeit darstellt, zeigen neue Analysen einer groß angelegten Studie mit Panik-Patienten (Gloster et al., persönliche Mitteilung).

Für die Psychotherapieforschung und -evaluation ist relevant, dass der FAH-II änderungssensitiv ist: Während sich in der Wartekontrollgruppe der Studie von Gloster et al. [2011b] keine Hinweise auf Veränderungen der PF ergaben, war sie am Ende einer Manual-basierten Verhaltenstherapie gestiegen (kontrollierte Effektstärke: d =0,72). Obwohl das Verfahren nicht für die Differenzialdiagnostik entwickelt wurde, so differenziert es doch zwischen Patienten und der Kontrollgruppe. Betrachtet man Angst-Patienten mit Panikstörung und Agoraphobie oder Sozialer Phobie (N = 577; M (Mittelwert $)=40,1 /$ SD (Standardabweichung) = 7,7) und klinisch unauffällige Probanden (Studenten/Arbeitslose, $\mathrm{N}=590 ; \mathrm{M}=$ 33,3 / SD = 9,2), so ergeben Receiver Operating Characteristic (ROC)-Analysen einen FAH-II-cut-off-Wert von 40 (Area under the curve $(\mathrm{AUC})=0,716 ; 95 \%$ Konfidenzintervall (CI): 0,682-0,750).

Errechnet man aus den Prä- und Post-Werten von N = 301 Panik-Patienten [Gloster et al., 2011b], wie groß der Unterschied zwischen 2 PF-Messwerten werden muss, um auf dem 95\%-Niveau klinisch signifikant zu sein [Hoyer et al., 2008, S. 383], so ergibt sich ein Wert von 7 (95\% CI 1,31-12,84).
Die zahlreichen, mit der Originalversion [Bond et al., 2011] und der deutschen Version [Gloster et al., 2011a] gewonnenen Hinweise auf die Reliabilität und Validität des Fragebogens, insbesondere diejenigen zur inkrementellen Validität und zur Änderungssensitivität, sprechen für seinen Einsatz in der Forschung. Auch wenn für die deutsche Fassung im Moment nur Daten aus dem Bereich der Angstbehandlung vorliegen, so ist auf die internationalen Publikationen [Bond et al., 2011] zu verweisen, die nahe legen, dass das Verfahren unter transdiagnostischer Perspektive eingesetzt werden kann.

Im Hinblick auf die Fragebogenmethode gilt aber kritisch zu berücksichtigen, dass PF eigentlich eine dynamische Interaktion zwischen Individuum, seinen Emotionen und seinen Handlungen beschreibt und deswegen am besten mit experimentellen Anordnungen oder mittels ExperienceSampling-Methoden zu untersuchen wäre. PF mit Selbstaussagen zu erfassen, kann nur ein erster, besonders ökonomischer Schritt sein [Kashdan und Rottenberg, 2010]. Ebenfalls kritisch ist zu werten, dass die Items des Fragebogens nicht bezogen auf die Fähigkeit der PF formuliert wurden, sondern inhaltlich eher psychologische Inflexibilität bezeichnen. Dies wurde aber bewusst übernommen, da die hier vorgestellte Version zum Ziel hat, die internationale Vergleichbarkeit deutschsprachiger Untersuchungen vor einem ACT-Hintergrund zu unterstützen. Dabei ist kritisch darauf verwiesen, dass die spezifische inhaltliche Validität bei etlichen Items nur bedingt erkennbar ist, da die Items auch schlicht als globale Indikatoren psychopathologischer Beeinträchtigung verstanden werden können: Das Item «Die Sorgen stellen sich meinem Erfolg in den Weg» beispielsweise könnte auf den ersten Blick auch ein Symptomindikator für Depression oder Generalisierte Angststörung sein. Aus der ACT-Perspektive ist dieses Item aber deswegen geeignet, weil (theoretisch) die PF darüber entscheidet, ob gegebene Sorgen zu Einschränkungen führen (müssen), oder ob sie akzeptiert werden können (was mit weniger Einschränkungen verbunden wäre). Auch ein Respondent mit sehr vielen Sorgen könnte auf das Item mit «nein» antworten: er wäre dann «psychologisch flexibel».

Eine für die Verhaltenstherapie wichtige Frage ist, ob der Fragebogen sich auch eignet, um Therapieverläufe über eine reine Störungs- und Symptomdiagnostik hinaus zu evaluieren und zu dokumentieren [Hoyer und Uhmann, 2008] und damit anzuzeigen, dass die Therapie auch die PF gefördert hat; die gezeigten Befunde zur prädiktiven Validität und Änderungssensitivität sprechen vorläufig dafür. Allerdings ist die Änderungssensitivität geringer als bei störungsspezifischen Symptomskalen [Gloster et al., 2011a].

Die hier dargestellten Cut-off-Werte sollen erste Anhaltspunkte für den Einsatz des Verfahrens in der Thera- 
piepraxis liefern. Dabei gilt es, die Daten vorsichtig zu interpretieren, denn der Einsatz des Verfahrens in der Individualdiagnostik sollte nur im Kontext der gesamten vorliegenden, insbesondere verhaltensdiagnostischen Informationen erfolgen.

\section{Disclosure Statement}

Die Autoren erklären hiermit, dass keine Interessenskonflikte vorliegen. Jürgen Hoyer erhielt Vortragshonorare von Astra-Zeneca, Andrew T. Gloster von Pfizer, ohne dass ein Zusammenhang mit den hier vorgelegten Daten besteht. Die Autoren bedanken sich für die finanzielle Unterstützung durch das Bundesministerium für Bildung und Forschung (Projekt 01GV0615) und die Deutsche Forschungsgemeinschaft (HO1900/5-1).

\section{Literatur}

Bond FW, Hayes SC, Baer RA, Carpenter KC, Guenole N, Orcutt HK, Waltz T, Zettle RD: Preliminary psychometric properties of the acceptance and action questionnaire - II: a revised measure of psychological flexibility and acceptance. Behav Ther 2011;42:676-688

Eifert GH: Akzeptanz- und Commitment-Therapie. Göttingen, Hogrefe, 2011.

- Gloster AT, Klotsche J, Chaker S, Hummel KV, Hoyer J: Assessing psychological flexibility: what does it add above and beyond existing constructs? Psychol Assess 2011a;4:970-982.
Gloster AT, Wittchen H-U, Einsle F, Lang T, HelbigLang S, Fydrich T, Fehm L, Hamm AO, Richter J, Alpers GW, Gerlach AL, Ströhle A, Kircher T, Deckert J, Zwanzger P, Höfler M, Arolt V: Psychological treatment for panic disorder with agoraphobia: a randomized controlled trial to examine the role of therapist-guided exposure in situ in CBT. J Consult Clin Psychol 2011b;79:406-420.

Hayes SC, Strosahl KD, Wilson KG, Bissett RT, Pistorello J, Toarmino D, Polusny MA, Dykstra TA, Batten SV, Bergan J, Stewart SH, Zvolensky MJ, Eifert GH, Bond FW, Forsyth JP, Karekla M, McCurry S: Measuring experiential avoidance: a preliminary test of a working model. Psychol Rec 2004; 54:553-578.

Hayes SC, Strosahl KD, Wilson KG: Acceptance and Commitment Therapy: The Process and Practice of Mindful Change, ed 2. New York, Guilford Press, 2012.
Hoyer J, Margraf J, Schneider S: Fragebogen, Ratingskalen und Tagebücher für die verhaltenstherapeutische Praxis; in Margraf J, Schneider S (eds): Lehrbuch der Verhaltenstherapie, ed 3. Heidelberg, Springer, 2008, pp 377-390.

Hoyer J, Uhmann S: Therapieevaluation. Klinische Diagnostik und Evaluation 2008;1:84-106.

Kashdan TB, Rottenberg J: Psychological flexibility as a fundamental aspect of health. Clin Psychol Rev 2010;30:865-878.

Luoma B, Hayes SC, Walser RD: ACT-Training Handbuch der Acceptance \& Commitment Therapie. Paderborn, Junfermann, 2009. 